\title{
Technique for Proficiently Yielding Deep-Web Interfaces using Smart Crawler
}

\author{
Devendra Hapase \\ Me Computer (Engineering), \\ Jayawantrao Sawant College of Engineering, \\ Hadapsar, Pune-28, Savitribai Phule Pune \\ University, Pune, India
}

\author{
M. D. Ingle \\ Asst. Prof (Computer Engineering), \\ Jayawantrao Sawant College of Engineering, \\ Hadapsar, Pune-28, \\ Savitribai Phule Pune \\ University, Pune, India
}

\begin{abstract}
Now days, world web has most famous because of web as well as internet increased development and its effect is that there are more requirements of the techniques that are used to improve the effectiveness of locating the deep-web interface. A technique called as a web crawler that surfs the World Wide Web in automatic manner. This is also called as Web crawling or spidering. In proposed system, initial phase is Smart Crawler works upon site-based scanning for mediatory pages by implementing search engines. It prevents the traffic that colliding with huge amount of pages. Accurate outcomes are taken due to focus upon crawl. Ranking of websites is done on the basis of arrangements on the basis of the priority valuable individuals and quick in-site finding through designing most suitable links with an adaptive link-ranking. There is always trying to search the deep web databases that doesn't connected with any of the web search tools. They are continuous insignificantly distributed as well as they are constantly modifying. This issue is overcome by implementing two crawlers such as generic crawlers and focused crawlers. Generic crawlers aggregate every frame that may be found as well as it not concentrate over a particular subject. Focused crawlers such as Form-Focused Crawler (FFC) and Adaptive Crawler for Hidden-web Entries (ACHE) may continuous to find for online databases on a specific subject. FFC is designed to work with connections, pages as well as from classifiers for focused crawling of web forms and it is extended through adding ACHE with more components for filtering and adaptive link learner. This system implements Naive Bayes classifier instead of SVM for searchable structure classifier (SFC) and a domain-specific form classifier (DSFC). Naive Bayes classifiers in machine learning are a bunch of clear probabilistic classifiers determine by implementing Bayes theorem with solid (gullible) freedom assumptions from the components. The proposed system contribute a novel module user login for selection of authorized user who may surf the particular domain on the basis of provided data the client and that is also used for filtering the results. In this system additionally implemented the concept of pre-query as well as post-query. Pre-query works only with the form and with the pages that included it and Post-query is utilizes data collected outcomes from form submissions.
\end{abstract}

\section{Keywords}

Deep web, crawler, feature selection, ranking, adaptive learning, Web resource discovery.

\section{INTRODUCTION}

In data age, most preferred thing is absolutely data. Data is essential requirement similar as food, shelter and clothing. Huge scale data is available over the web due to innovative developments that has becomes a difficult entity having data from the different sources. Different types of web searching tools are used to find out the data. A web seeker has permission to access a large data. But it still far from the treasury of information lying under the Web, an unlimited store of information past the compass of routine web crawlers: the "Deep Web" or "Invisible Web".

The components of the Deep Web are avoided up in the query outcomes of existing web crawlers. The crawlers of previous web crawlers recognize simply static pages and can't take to the dynamic Web pages of Deep Web databases. In this manner, the Deep Web is then again termed the "Hidden" or "Invisible Web". The term Invisible Web was organized by Dr. Jill Ellsworth to allude to information hard to reach to previous web crawlers. In any case, using the term Invisible Web to depict recorded information that is open however not easily accessible which is not exact.

In previous framework to locate the deep web databases is a threat, since they are not enrolled with any web search tools are regularly pitifully scattered and hold always showing signs of change. Thus proposing Naive Bayes classifier variant of SVM classifier for searchable form classifier (SFC) and a domain specific form classifier (DSFC). In machine learning, Naive Bayes classifiers are a bunch of fundamental probabilistic classifiers taking into account implementing Bayes theorem with solid (naive) autonomy suspicions within the attributes. Nave bays are quick and space effective, not touchy to insignificant attributes and manage Streaming information effectively.

System also contributes a new module on the basis of user login for selected registered users who can surf the particular domain regarding provided input by the user. This module is additionally utilized for filtering the outcomes.

\subsection{Contribution Work}

The proposed system has able to implement for focused crawler. Contribution gives the huge coverage and obtains the high effectiveness in focused crawler.

Contribution 1: Proposing Naive Bayes classifier rather than SVM for searchable form classifier (SFC) and a domainspecific form classifier (DSFC).

\section{Advantages:}

- Naive bayes is quick and effective in utilizing space.

- Not sensitive to irrelevant aspects.

- Handles Streaming data effectively.

Contribution 2: System contributing new module depending over user login for selected registered clients who can surf the 
particular domain agreeing to given input by the user. This is module is additionally utilized for filtering the outcomes.

Contribution 3: The Third contribution is about pre-query \& post query. Pre-Query recognizes web databases through analyzing the huge variety in content and structure of forms.

The paper talks about Literature Survey on topic in Section 2. Section 3 provides System Overview, Mathematical Model, Current implementation details, Algorithm used, complexity Analysis. Section 4 shows Results and where as in Section 5 conclusions and presents future work are given.

\section{LITERATURE REVIEW}

In this section work done by the researchers for crawling process is discussed.

Feng Zhao, Jingyu Zhou, Chang Nie, Heqing Huang, Hai Jin [1] provides a two-stage system, for the Smart Crawler, for efficient aggregating deep web interfaces. Initially, Smart Crawler performs site-based searching for focused pages with the help of search engines, holding up from going to an extensive quantity of pages.

Soumen Chakrabarti, Martin van den Berg, Byron Dom [2] developed two hypertext mining projects, that classifier assess the frequency of a hypertext report as for the focus themes and distiller that indentifies hypertext nodes. Hypertext nodes are special access focuses to different particular pages within of a few joins.

Kevin Chen-Chuan Chang, Bin He, Chengkai Li, MiteshPatel, and Zhen Zhang presented this paper [3] for calculating appropriate features, analyzing as well as associating arranged Web sources to expanded not analyzed limit. Whole analysis provides deep data of Web and taking the dictatorial IP testing approach with one million tests and lower study provides source-regarding features more than 441 sources in eight representative domains.

Soumen Chakrabarti, Kunal Punera and Mallela Subramanyam [4] illustrates there is a large amount of important information over a HREF source page regarding the centrality of the objective page. The information is encrypted in appropriated manner and misused by an organized learner getting online practice from a customary focused crawler by observing an accurately arranged order of components and events regard with the crawler.

Sriram Raghavan and Hector Garcia-Molina [5] concentrated over the problem of developing a crawler efficient of dividing contents from this hidden Web. A common operational model developed at Stanford, of a Web crawler and illustrates how demonstrated model is identified in HiWE (Hidden Web Exposer).

Jayant Madhavan, Shawn R. Jeffery, Shirley Cohen, XinDong, David Ko, Cong Yu, andAlon Halevy [6] concentrated over complexities inside two circumstances such as the deep Web and Google Base. Authors accomplish that customary data association approaches are no more important with scale as well as heterogeneity.

Jared Cope, Nick Craswell and David Hawking describes [7] web internet seekers function but not to search datasets breach behind Web search frames that are appropriate to search crawlable pages. New technique for identifying search frames wills the base for a recent distributed search application.

Thomas Kabisch, Eduard C. Dragut, Clement Yu, and Ulf Leser [8] represent VisQI (Visual Query interface Integration framework) and a Deep Web integration system. VisQI may do (1) modifying Web query interfaces inside hierarchically arranged representations, (2) classify them within application domains and (3) associating the elements of different interfaces.

\section{IMPLEMENTATION DETAILS 3.1 Problem Definition}

There is main issue of the extensive size of web assets, frequent modifying behavior of deep web, taking wide coverage and high effectiveness. As deep web creates at a quick pace, there has been broadened energy for systems that help capably locate deep-web interfaces. In any case, in view of the incomprehensible volume of web resources and the dynamic method for deep web, achieving wide attraction and high effectiveness is a major issue. This paper proposes a successful deep web harvesting system, specifically Smart Crawler, for achieving both wide scope and high efficiency for a focused crawler.

\subsection{System Overview}

Figure 1 demonstrates the architectural view of the proposed system. The description of the system is as follows:

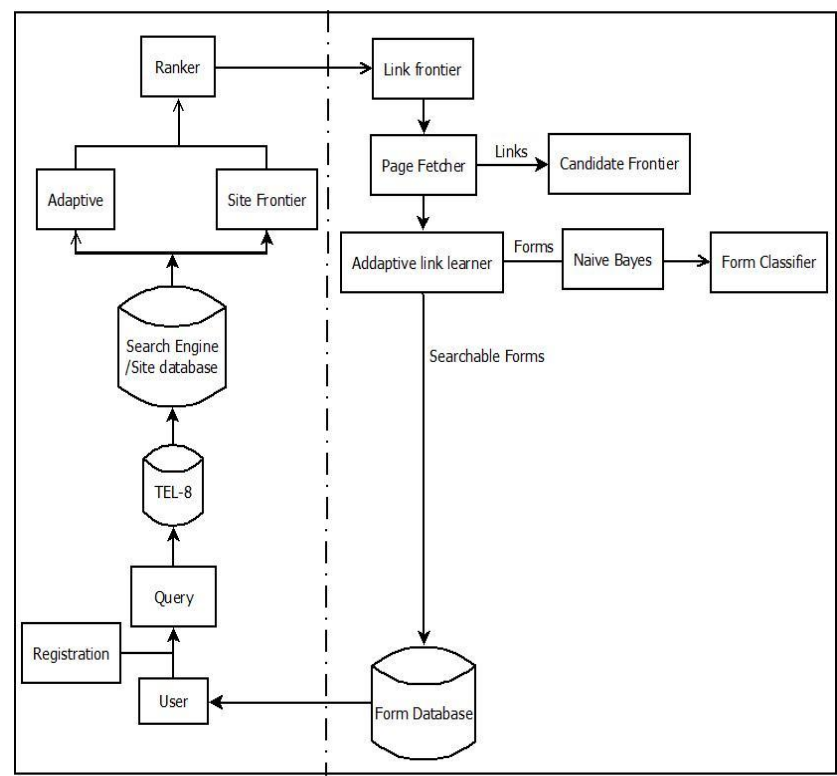

Fig 1: System Architecture

In proposed system, user gives a search query as an input. Then system started search on the basis of query in offline database of the system. If there is no results are found then system goes to online search on Google search engine. System also specifies the limit by threshold value to search results.

Online searched sites are stored and that are called as seed sites. In that searched sites once again reverse search is applied to find out the links-of-links. Over that links Naïve Bayes classifier is implemented for to classify the links or sites. After, on the basis of relevancy, ranking of links or sites are accomplished. Then graphs are generated by implementing SVM as well as Naïve Bayes Classifiers. In this system our contribution is Naïve Bayes classifier.

System is implemented module wise in following ways:

Module 1: 
- Seed Sites: Seeds sites are applicant sites given for Smart Crawler to start crawling.

- Site Database: Site database contains collection of web links or sites inside the database.

- Reverse Searching: At the point when the various unvisited URLs in the database is not exactly as a threshold at the time the crawling method.

Module 2:

- Site Frontier: Site Frontier retrieves homepage URLs from the site database.

- $\quad$ Adaptive Site Learner: The Site Ranker is improved during crawling by an Adaptive Site Learner.

- Site Ranker: In Smart Crawler, Site Ranker relegates a score for every site which is not visited that is related to its relevance to the already found deep web sites.

- Site Classifier: The high priority queue is for out-ofsite links that are classified as relevant by Site Classifier and are judged by Form Classifier to contain searchable forms.

Module 3:

- Link Frontier: Links of a site are stored in Link Frontier and related pages are fetched and added forms are consolidated by Form Classifier to find searchable forms.

- Link Ranker: Link Ranker organizes connects so that Smart Crawler can rapidly find searchable structures.

- $\quad$ Page Fetcher: Page Fetcher directly fetch out center page of the web site.

- Candidate Frontier: The links in web pages are brought into Candidate Frontier.

Module 4

- Form Classifier: Classifying forms plans to keep structure focused crawling, which sift through nonsearchable and unessential forms.

- $\quad$ Adaptive Link Learner: The Link Ranker is by ad improved by an Adaptive Link Learner, which gains from the URL way leading applicable forms.

- Form Database: Form database contains collection of sites; it collects all data which got input from Form Classifier.

Module 5

- Proposing new classifier Naive Bayes instead of SVM for searchable form classifier (SFC) and a domain-specific form classifier (DSFC).

\subsection{Mathematical Model}

System $S$ is defined as

$\mathrm{S}=\{\mathrm{LP}, \mathrm{I}, \mathrm{R}, \mathrm{SR}, \mathrm{SC}, \mathrm{LF}, \mathrm{FP}, \mathrm{P}, \mathrm{O}\}$

\section{Input :}

Login Process

$\mathrm{LP}=\{\operatorname{lp} 1, \mathrm{lp} 2, \ldots, \mathrm{lpn}\}$

Where, LP is the set of login users and

$1 \mathrm{p} 1,1 \mathrm{p} 2, \mathrm{lp} 3, \ldots . ., \mathrm{lpn}$ are the number of users.

Query

$\mathrm{I}=\{\mathrm{i} 1, \mathrm{i} 2, \ldots, \mathrm{in}\}$
Where, $I$ is the set of queries and i1, i2, i3, .....,in are the number individuals query.

2. Process :

- $\quad$ Reverse Search

$$
\mathrm{R}=\{\mathrm{A} ; \mathrm{S}\}
$$

Where, $\mathrm{R}$ is represent as a Reverse Search in which content $\mathrm{A}=$ Adaptive Learning, $\mathrm{S}=$ Site Frontier

- $\quad$ Site Ranking

$$
\mathrm{SR}=\{\mathrm{sr} 1, \mathrm{sr} 2, \ldots, \mathrm{srn}\}
$$

Where SR is the set of Site Ranking and sr1, sr2, sr3, ....., srn represent as a number of rank site.

Site ranking Rank(s) is obtained by following formula, which is the function of site similarity $\mathrm{ST}(\mathrm{s})$ and site frequency $\mathrm{SF}(\mathrm{s})$.

$$
\begin{gathered}
\operatorname{Rank}(\mathrm{s})=\mathrm{ST}(\mathrm{s})+\mathrm{SF}(\mathrm{s})(1) \\
\mathrm{ST}(\mathrm{s})=\operatorname{Sim}(\mathrm{U}, \mathrm{Us})+\operatorname{sim}(\mathrm{A}, \mathrm{As})+\operatorname{sim}(\mathrm{T}, \mathrm{Ts})(2)
\end{gathered}
$$

Where, Sim calculate the similarity between features of s.

$$
\operatorname{Sim}(V 1, V 2)=\frac{V 1 . V 2}{|V 1| *|V 2|}
$$

SF calculates the number of times site appear in other site.

$$
S F(S)=\sum_{\text {knownsiteslist }} l t
$$

- $\quad$ Site Classifier

$$
\mathrm{SC}=\{\mathrm{sc} 1, \mathrm{sc} 2, \ldots, \mathrm{scn}\}
$$

Where SC is the set of Site Classifier and sc1, sc2,sc3, .....,scn represent as a number of classified site.

- $\quad$ Link Frontier

$$
\mathrm{LF}=\{1 \mathrm{f} 1,1 \mathrm{f} 2, \ldots, \mathrm{lfn}\}
$$

Where LF is the set of Link Frontier andlf1, lf2, lf3, .....,lfn represent as a number of frontier link.

- $\quad$ Fetch Pages

$$
F P=\{f p 1, f p 2, f p 3, \ldots, f p n\}
$$

Where, FP is the set of Fetch Pages andfp1, fp2,fp3, ....fpn are the number of pages which are fetch.

- $\quad$ Link Ranking

$$
\begin{aligned}
& \mathrm{L}=\{11,12, \ldots, \ln \} \\
& \text { Where } \mathrm{L} \text { is the set of all ranked links. }
\end{aligned}
$$

$$
\mathrm{LT}(\mathrm{l})=\operatorname{Sim}(\mathrm{P}, \mathrm{P} 1)+\operatorname{sim}(\mathrm{A}, \mathrm{Al})+\operatorname{sim}(\mathrm{T}, \mathrm{Tl})
$$

- $\quad$ Pre-query and Post-query

$$
\mathrm{P}=\{\mathrm{P} 1, \mathrm{P} 2\}
$$

Where, $\mathrm{P}$ is represent as a Pre-query and Post-query in which content $\mathrm{P} 1=$ Prequery, $\mathrm{P} 2=$ Postquery.

3. Output

Searchable Form $\mathrm{O}=\{\mathrm{o} 1, \mathrm{o} 2, \mathrm{o} 3, \ldots$, on $\}$

Where, $\mathrm{O}$ is the set of Searchable Form and o1, o2, o3, ....on are the number of searchable form.

\subsection{Algorithm Used}

\section{Algorithm 1: Proposed Algorithm}

Input: Query

Output: Searchable and domain specific links 


\section{Process:}

1: User login to the system

2: Preprocessing of user query

3: while(no. of sites < threshold)

4: do

site $=$ fetchDeepSites () ;

link = reverceSearch $($ site $)$;

for all link in links

\section{\{}

text $=$ extractPageData(link)

relevantSite $=\operatorname{classify}($ text $)$;

sitefrontier $=$ relevantSite;

\}

end

5: while(sitefrontier not null)

do

link $=$ sitefrontier.getlink ()

relevant $=$ classifylink $($ link $)$

if(relevant $)\{$

output $=$ searchable and domain specific forms

\}

postquery processing an output

end

Algorithm 2: Naive Bayes

Input: arff file

Output: Classification of instances

\section{Process:}

1. Frequency Table is created by conversion of data set.

2. Likelihood table is created by finding the probabilities like Overcast probability $=0.29$ and probability of playing is 0.64 .

3. After that, use Naive Bayesian equation to compute the posterior probability for each class. The output of prediction is the class with the major posterior.

\subsection{Complexity Analysis}

- FOR SVM:

$$
\mathrm{O}(\mathrm{n} 2) \text { and } \mathrm{O}(\mathrm{n} 3)
$$

- FOR C45:

$$
\mathrm{O}\left(\mathrm{m}^{*} \mathrm{n}\right) 2+\mathrm{O}\left(\mathrm{m}^{*} \mathrm{n}\right)
$$

- For Naive Byes:

$$
\mathrm{O}(\mathrm{m} * \mathrm{n})
$$

- $\quad$ Overall time required:

$$
\mathrm{O}(\mathrm{m} * \mathrm{n})+\mathrm{O}(\mathrm{m} * \mathrm{n}) 2+\mathrm{O}(\mathrm{m} 3)
$$

Where,

$\mathrm{m}=$ number of training instance

$\mathrm{n}=$ number of attributes $\mathrm{s}$

\subsection{Experimental Setup}

For implementation system required JDK 1.8and netbeans 1.8 development tool.

\section{RESULT AND DISCUSSION}

\subsection{Dataset Discussion}

TEL-8 dataset is utilized that is accessed from the UCI repository. Classifier trained the information by utilizing this dataset. As a source can contain number of interfaces, the TEL-8 dataset has 447 deep web sources with 477 query interfaces.

\subsection{Results}

Table 1 demonstrates the outcomes of accuracy of site classifier and form classifier obtained by proposed system and existing system.

Table 1. Accuracy Comparison Table

\begin{tabular}{|c|c|c|}
\hline Classifier & $\begin{array}{c}\text { System with SVM } \\
\text { Classifier }\end{array}$ & $\begin{array}{c}\text { System with Naïve } \\
\text { Bayes Classifier }\end{array}$ \\
\hline Summary & $78 \%$ & $90 \%$ \\
\hline
\end{tabular}

Fig. 2 demonstrates the comparison between accuracy of proposed system and existing system. The proposed system is more accurate compared with the existing system.

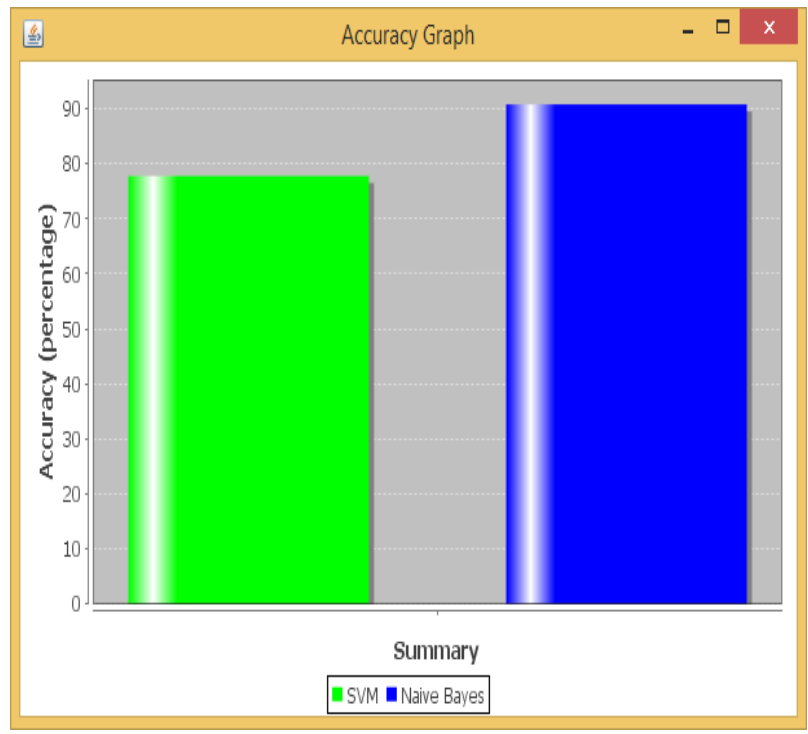

Fig 2: Accuracy Graph

Table 2 demonstrates the outcomes for time needed for utilizing site classifier and form classifier for proposed system and existing system.

Table 2. Time Comparison table

\begin{tabular}{|c|c|c|}
\hline Classifier & $\begin{array}{c}\text { System with SVM } \\
\text { Classifier }\end{array}$ & $\begin{array}{c}\text { System with Naïve } \\
\text { Bayes Classifier }\end{array}$ \\
\hline Time in Nanosec & $14,000,000$ & $10,005,000$ \\
\hline
\end{tabular}

Fig. 3 demonstrates the time comparison between the existing and proposed system. 


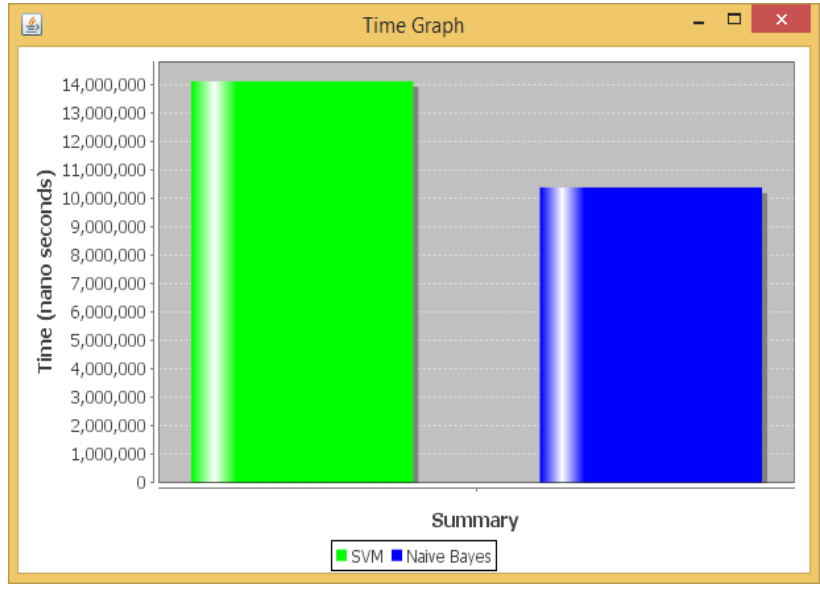

Fig 3: Time Graph

\section{CONCLUSION AND FUTURE SCOPE}

There is a problem to locate the particular web databases, in case of that they are not connected with any of the search engines and also distributed as well as frequently modifying. To overcome this issue, this paper introduces an efficient harvesting system for deep-web interfaces that is also called as Smart-Crawler. This system present the strategy to solves both large area for deep web interfaces and also provide more effective crawling. On the basis of rank based aggregated sites as well as focused the crawling over a topic, Smart Crawler achieves more accurate outcomes. Adaptive link-ranking is implemented to search a site within in-site exploring stage and also generate a link tree for destroy bias for particular directories of a site for extra expansive scope of web directories. Experimental outcomes over a dataset of domains are shows the efficiency of proposed two-stage crawler that provides higher harvest rates as compared with other. This system utilized a novel classifier Naive Bayes rather than SVM for searchable form classifier (SFC) and a domainspecific form classifier (DSFC). This system contributes a new module client login to select registered users who can surf the specific domain as shown via provided input by the user. Outcomes are also filtered by using this module.

In further work have tendency to join pre-query and postquery methodologies for ranking deep web forms to further enhance the correctness to the form classifier.

\section{REFERENCES}

[1] Feng Zhao, Jingyu Zhou, Chang Nie, Heqing Huang, Hai Jin, SmartCrawler: A Two-stage Crawler for Efficiently Harvesting Deep-Web Interfaces in IEEE Transactions on Services Computing Volume: PP Year: 2015.

[2] Soumen Chakrabarti, Martin van den Berg, Byron Dom, and Focused crawling: a new approach to topic-specific $\begin{array}{llll}\text { Web } & \text { resource } & \text { discovery, } & \text { Computer } \\ \text { Networks,31(11):16231640, } & 1999 .\end{array}$

[3] Kevin Chen-Chuan Chang, Bin He, Chengkai Li, MiteshPatel, and Zhen Zhang. Structured databases on the web: Observations and implications. ACM SIGMOD Record, 33(3):61-70, 2004.

[4] Soumen Chakrabarti, Kunal Punera and Mallela Subramanyam. Accelerated focused crawling through online relevance feedback. In Proceedings of the 11th international conference on World Wide Web, pages 148-159, 2002.

[5] Sriram Raghavan and Hector Garcia-Molina. Crawling the hidden web. In Proceedings of the 27th International Conference on Very Large Data Bases, pages 129-138, 2000 .

[6] Jayant Madhavan, Shawn R. Jeffery, Shirley Cohen, XinDong, David Ko, Cong Yu, andAlon Halevy. Webscale data integration: You can only afford to pay as you go. In Proceedings of CIDR, pages 342-350, 2007.

[7] Jared Cope, Nick Craswell and David Hawking. Automated discovery of search interfaces on web. In Proceedings of the 14th Australasian database conference- Volume 17, pages 181-189. Australian Computer Society, Inc., 2003.

[8] Thomas Kabisch, Eduard C. Dragut, Clement Yu, and Ulf Leser. Deep web integration with visqi. Proceedings of the VLDB Endowment, 3(1-2):1613-1616,2010

\section{AUTHOR PROFILE}

Mr. Devendra S. Hapase, is currently pursuing M.E (Computer) from Department of Computer Engineering, JayawantraoSawant College of Engineering, Pune, India. SavitribaiPhule Pune University, Pune, Maharashtra, India 411007. He received his B.E (Computer) Degree from SKNCOE, Pune, India. SavitribaiPhule Pune University, Pune, Maharashtra, India -411007. His area of interest is network security and web\& data mining.

Prof. M.D Ingle, received his M Tech. (Computer) Degree from Dr. BabasahebAmbedkar Technological University, Lonere, Dist. Raigad-402 103, Maharashtra, India. He received his B.E (Computer) Degree from Govt college of Engineering, Aurangabad, Maharashtra, India.He is currently working as M.E coordinator and Asst Prof (Computer) at Department of Computer Engineering, JayawantraoSawant College of Engineering, Pune, India. SavitribaiPhule Pune University, Pune, Maharashtra, India -411007 His area of interest is network security and web\&data mining. 